\title{
Intravesical Bacillus Calmette-Guérin versus mitomycin C for Ta and T1 bladder cancer: Abridged summary of the Cochrane Review
}

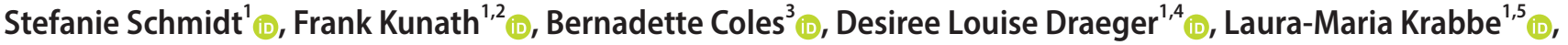

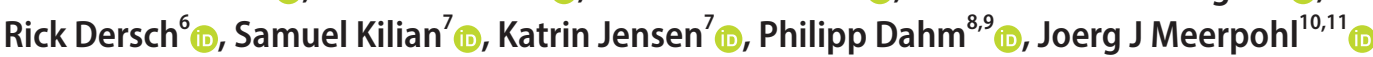 \\ 'UroEvidence@Deutsche Gesellschaft für Urologie, Berlin, ²Department of Urology, University Hospital Erlangen, Erlangen, Germany, ${ }^{3}$ Velindre NHS Trust, Cardiff \\ University Library Services, Cardiff, United Kingdom, ${ }^{4}$ Department of Urology, University of Rostock, Rostock, ${ }^{5}$ Department of Urology, University of Muenster Medical \\ Center, Muenster, ${ }^{6}$ Clinic of Neurology and Neurophysiology, Medical Center- University of Freiburg, Faculty of Medicine, University of Freiburg, Freiburg, Institute of \\ Medical Biometry and Informatics, University of Heidelberg, Heidelberg, Germany, ${ }^{8}$ Urology Section, Minneapolis VA Health Care System, Minneapolis, MN, ${ }^{9}$ Department \\ of Urology, University of Minnesota, Minneapolis, MN, USA, ${ }^{10}$ Institute for Evidence in Medicine, Medical Center - University of Freiburg, Faculty of Medicine, University of \\ Freiburg, Freiburg, "Cochrane Germany, Cochrane Germany Foundation, Freiburg, Germany
}

Keywords: Administration, intravesical; BCG vaccine; Mitomycin; Systematic review; Urinary bladder neoplasms

This is an Open Access article distributed under the terms of the Creative Commons Attribution Non-Commercial License (http://creativecommons.org/licenses/by-nc/4.0) which permits unrestricted non-commercial use, distribution, and reproduction in any medium, provided the original work is properly cited.

\section{INTRODUCTION}

People with urothelial carcinoma of the bladder are at risk for recurrence and progression following transurethral resection of a bladder tumour. Mitomycin C (MMC) and Bacillus Calmette-Guérin (BCG) are commonly used, competing forms of intravesical therapy for intermediate- or high-risk non-muscle invasive ( $\mathrm{Ta}$ and $\mathrm{T} 1$ ) urothelial bladder cancer but their relative merits are somewhat uncertain. Although several systematic reviews and meta-analyses have been conducted on this topic, it still remains unclear what the optimal treatment dose and schedule might be, as well as the question of which people benefit most from one or the other agent $[1,2]$.

\section{Objectives}

We assessed the effects of MMC compared to BCG for treating intermediate- or high-risk non-muscle invasive urothelial bladder cancer.

\section{MATERIALS AND METHODS}

We updated a previously published Cochrane Review to assess the effects of MMC compared to BCG by searching systematically and comprehensively the biomedical literature in multiple databases (CENTRAL, MEDLINE, EMBASE, Web of Science, Scopus, LILACS, ClinicalTrials. gov, World Health Organization International Clinical Trials Registry Platform) up to 23th September 2019 [3]. Supplementary material 1 shows the search strategies. We also hand searched the reference lists of included articles as well

\section{Received: 15 April, 2020 • Accepted: 3 May, 2020}

Corresponding Author: Stefanie Schmidt (iD https://orcid.org/0000-0002-0868-318X

See Editorial on page 353

Deutsche Gesellschaft für Urologie e.V., Martin-Buber-Str. 10, 14163 Berlin, Germany

TEL: +49-30-887083312, FAX: +49-211-51609660, E-mail: schmidt@dgu.de

This article is based on a Cochrane Review published in the Cochrane Database of Systematic Reviews (CDSR) 2020, Issue 1, DOI: 10.1002/14651858. CD011935.pub2. Cochrane Reviews are regularly updated as new evidence emerges and in response to feedback, and the CDSR should be consulted for the most recent version of the review. 
as conference proceedings. We did not restrict by publication language or publication status.

We included randomised or quasi-randomised controlled trials comparing MMC to BCG for the treatment of nonmuscle invasive (Ta and $\mathrm{T} 1$ ) urothelial bladder cancer in adults. Neither sequential administration of BCG and MMC nor electromotive or hyperthermic drug stimulation were the focus of this review. Two independent reviewers screened identified references, extracted data, and assessed the risk of bias according to Cochrane's methodological recommendations [4].

We performed meta-analyses using the random effects model and assessed the heterogeneity between studies with the $\mathrm{I}^{2}$ statistic. All analyses were conducted with Review Manager 5 software [5]. We used the Grading of Recommendations, Assessment, Development, and Evaluation (GRADE) approach to rate the certainty of the evidence for each predefined outcome [4].

\section{RESULTS}

The literature search identified 1,125 records, of which 12 studies fulfilled our inclusion criteria (based on 29 publications, including 2,932 patients, published between 1995 and 2013). Eleven were included in the meta-analyses [6-16]. The one study that was not included in the meta-analysis was only available as a conference proceeding, which did not provide sufficient data for inclusion in the analysis [17].

Table 1 presents the summary of findings of the main outcomes [8,13]. Supplementary Table 1 and Supplementary Fig. 1 summarises the characteristics and the risk of bias of the included studies. Supplementary material 2 lists the excluded studies and the rationale for their exclusion.

\section{Primary outcomes}

We found low-certainty evidence that BCG may make little or no difference on time to death from any cause compared to MMC (hazard ratio [HR], 0.97; 95\% confidence interval [CI, 0.79 to 1.20; participants $=1,132$; studies $=5 ; 567$ participants in the BCG arm and 565 in the MMC arm; $\mathrm{I}^{2}=0 \%$ ). We also found low-certainty evidence that BCG may increase the risk for serious adverse effects compared to MMC (risk ratio, 2.31; 95\% CI, 0.82 to 6.52 ; participants $=1,024$; studies $=5$; 577 participants in the BCG arm and 447 in the MMC arm; $\mathrm{I}^{2}=0 \%$.

\section{Secondary outcomes}

We found low-certainty evidence that BCG may reduce the time to recurrence compared to MMC (HR, 0.88; 95\% CI,
0.71 to 1.09; participants $=2,616$; studies $=11 ; 1,273$ participants in the BCG arm and 1,343 in the MMC arm; $\mathrm{I}^{2}=61 \%$ ). Certainty of the evidence was also rated as low for time to progression, where BCG may make little or no difference compared to MMC (HR, 0.96; 95\% CI, 0.73 to 1.26; participants=1,622; studies $=6 ; 804$ participants in the BCG arm and 818 in the MMC $\left.\operatorname{arm} ; I^{2}=0 \%\right)$. There were no data on quality of life.

The visual test for publication bias did not indicate any important asymmetry. The subgroup analysis showed that higher versus lower doses of BCG resulted in higher rates of serious adverse effects when compared to MMC. We were unable to assess treatment effects between intermediate and high-risk groups due to lack of data. A sensitivity analysis based on studies with low risk of bias could not be performed due to lack of low risk studies.

\section{DISCUSSION}

The first Cochrane Review on this topic was published in 2003, and included seven trials based on 1,901 participants [3]. This review update includes further five trials, which were published meanwhile. It now reflects also the current Cochrane methodology, which includes the certainty of the evidence assessment according to the GRADE approach.

BCG may reduce the risk of recurrence over time, while it may have no effect on either the risk of progression or risk of death from any cause over time. Instead, BCG may increase the risk of serious adverse effects. All findings are based on low certainty of the evidence.

The judgement of low certainty of the evidence for all outcomes in this review means that further research is very likely to have an important impact on the confidence in the estimates of effects and is likely to change the estimates.

Of the 12 identified studies, six were planned and conducted in the 1990s and do not meet 2019s methodological quality standards. Only one trial was conducted after 2010 but results of this trial have not been published yet. One trial (recruitment 2009 to 2012) was closed prior to finalisation due to a lack of accrual. Blinding of participants did not take place in any of the 12 trials. General concerns, which led to downgrading, were study limitations (performance bias and allocation concealment), wide CIs resulting in imprecision (possibility for either important benefit or large harm) and study heterogeneity.

BCG usage must be further studied to predict patients who respond most to BCG therapy, and to determine the optimal schedule and amount of BCG delivery per patient. High-quality randomised controlled trials in people with intermediate- and high-risk bladder cancer with adequate 


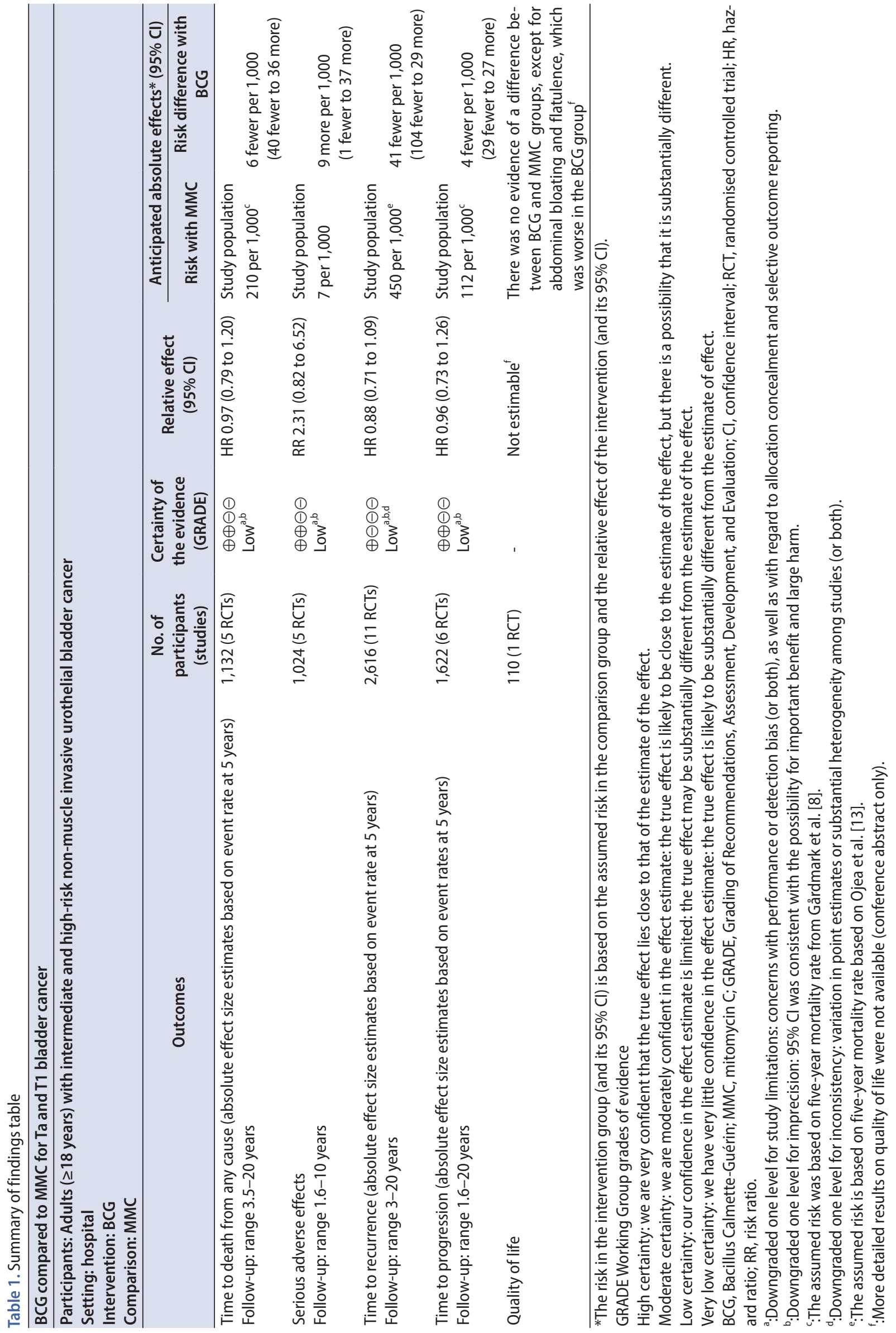


randomisation and blinding are warranted. They should address quality of life, adverse effects and time to progression to provide more reliable results for this patient population.

\section{CONCLUSIONS}

BCG may reduce the risk of recurrence over time, while it may have little or no effect on either the risk of progression or risk of death from any cause over time. However, BCG may increase the risk of serious adverse effects. All findings are based on low certainty of the evidence.

\section{CONFLICTS OF INTEREST}

The authors have nothing to disclose.

\section{ACKNOWLEDGMENTS}

We wish to thank the Cochrane Urology Group for their support and patience during this review process.

\section{AUTHORS' CONTRIBUTIONS}

Research conception and design: Stefanie Schmidt and Philipp Dahm. Data acquisition: Stefanie Schmidt, Rick Dersch, Desiree Louise Draeger, Laura-Maria Krabbe, and Bernadette Coles. Statistical analysis: Samuel Kilian and Katrin Jensen. Data analysis and interpretation: Stefanie Schmidt, Frank Kunath, and Joerg J Meerpohl. Drafting of the manuscript: Stefanie Schmidt and Joerg J Meerpohl. Critical revision of the manuscript: Frank Kunath and Philipp Dahm. Administrative, technical, or material support: Stefanie Schmidt and Bernadette Coles. Supervision: Philipp Dahm and Joerg J Meerpohl. Approval of the final manuscript: Stefanie Schmidt, Frank Kunath, Bernadette Coles, Desiree Louise Draeger, Laura-Maria Krabbe, Rick Dersch, Samuel Kilian, Katrin Jensen, Philipp Dahm, and Joerg J Meerpohl.

\section{SUPPLEMENTARY MATERIALS}

Scan this QR code to see the supplementary materials, or visit https:/www.icurology.org/src/sm/icurology-61-349-s001.pdf.

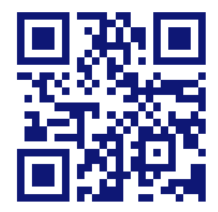

\section{REFERENCES}

1. Shelley MD, Mason MD, Kynaston H. Intravesical therapy for superficial bladder cancer: a systematic review of randomised trials and meta-analyses. Cancer Treat Rev 2010;36:195-205.

2. Böhle A, Jocham D, Bock PR. Intravesical bacillus CalmetteGuerin versus mitomycin C for superficial bladder cancer: a formal meta-analysis of comparative studies on recurrence and toxicity. J Urol 2003;169:90-5.

3. Shelley MD, Court JB, Kynaston H, Wilt TJ, Coles B, Mason M. Intravesical bacillus Calmette-Guerin versus mitomycin $\mathrm{C}$ for Ta and T1 bladder cancer. Cochrane Database Syst Rev 2003;(3):CD003231.

4. Higgins JPT, Green S (editors). Cochrane handbook for systematic reviews of interventions version 5.1.0 [Internet]. The Cochrane Collaboration; 2011 [updated 2011 Mar; cited 2019 Jul 19]. Available from: http://handbook.cochrane.org.

5. The Nordic Cochrane Centre. Review manager (RevMan). version 5.3 [Internet]. Copenhagen: The Nordic Cochrane Centre, The Cochrane Collaboration; 2014 [cited 2019 Jul 19]. Available from: https://training.cochrane.org/online-learning/coresoftware-cochrane-reviews/revman/revman-5-download.

6. Di Stasi SM, Giannantoni A, Stephen RL, Capelli G, Navarra $\mathrm{P}$, Massoud R, et al. Intravesical electromotive mitomycin C versus passive transport mitomycin $\mathrm{C}$ for high risk superficial bladder cancer: a prospective randomized study. J Urol 2003;170:777-82.

7. Friedrich MG, Pichlmeier U, Schwaibold H, Conrad S, Huland $\mathrm{H}$. Long-term intravesical adjuvant chemotherapy further reduces recurrence rate compared with short-term intravesical chemotherapy and short-term therapy with Bacillus CalmetteGuérin (BCG) in patients with non-muscle-invasive bladder carcinoma. Eur Urol 2007;52:1123-29.

8. Gårdmark T, Jahnson S, Wahlquist R, Wijkström H, Malmström PU. Analysis of progression and survival after 10 years of a randomized prospective study comparing mitomycin-C and bacillus Calmette-Guérin in patients with high-risk bladder cancer. BJU Int 2007;99:817-20.

9. Järvinen R, Kaasinen E, Sankila A, Rintala E. Long-term efficacy of maintenance bacillus Calmette-Guérin versus maintenance mitomycin $\mathrm{C}$ instillation therapy in frequently recurrent TaT1 tumours without carcinoma in situ: a subgroup analysis of the prospective, randomised FinnBladder I study with a 20year follow-up. Eur Urol 2009;56:260-5.

10. Krege S, Giani G, Meyer R, Otto T, Rübben H. A randomized multicenter trial of adjuvant therapy in superficial bladder cancer: transurethral resection only versus transurethral resection plus mitomycin $\mathrm{C}$ versus transurethral resection plus bacillus Calmette-Guerin. Participating Clinics. J Urol 1996;156:962-6. 
11. Lamm DL, Blumenstein BA, David Crawford E, Crissman JD, Lowe BA, Smith JA Jr, et al. Randomized intergroup comparison of bacillus calmette-guerin immunotherapy and mitomycin $\mathrm{C}$ chemotherapy prophylaxis in superficial transitional cell carcinoma of the bladder a southwest oncology group study. Urol Oncol 1995;1:119-26.

12. Mangiarotti B, Trinchieri A, Del Nero A, Montanari E. A randomized prospective study of intravesical prophylaxis in non-musle invasive bladder cancer at intermediate risk of recurrence: mitomycin chemotherapy vs BCG immunotherapy. Arch Ital Urol Androl 2008;80:167-71.

13. Ojea A, Nogueira JL, Solsona E, Flores N, Gómez JM, Molina $\mathrm{JR}$, et al. A multicentre, randomised prospective trial comparing three intravesical adjuvant therapies for intermediate-risk superficial bladder cancer: low-dose bacillus Calmette-Guerin (27 mg) versus very low-dose bacillus Calmette-Guerin (13.5 mg) versus mitomycin C. Eur Urol 2007;52:1398-406.

14. Witjes JA, v d Meijden AP, Collette L, Sylvester R, Debruyne FM, van Aubel A, et al. Long-term follow-up of an EORTC randomized prospective trial comparing intravesical bacille Calmette-Guérin-RIVM and mitomycin C in superficial bladder cancer. EORTC GU Group and the Dutch South East Cooperative Urological Group. European Organisation for Research and Treatment of Cancer Genito-Urinary Tract Cancer Collaborative Group. Urology 1998;52:403-10.

15. Witjes WP, Witjes JA, Oosterhof GO, Debruyne MJ. Update on the Dutch Cooperative Trial: mitomycin versus bacillus Calmette-Guérin-Tice versus bacillus Calmette-Guérin RIVM in the treatment of patients with pTA-pT1 papillary carcinoma and carcinoma in situ of the urinary bladder. Dutch South East Cooperative Urological Group. Semin Urol Oncol 1996;14(1 Suppl 1):10-6.

16. ClinicalTrials.gov. Mitomycin C Versus Bacillus CalmetteGuerin in the intravesical treatment of non-muscle-invasive bladder cancer patients: a randomized phase III non-inferiority trial [Internet]. Bethesda: National Library of Medicine; 2009 [cited 2019 Jul 19]. Available from: www.clinicaltrials.gov/ct2/ show/NCT00974818.

17. Michielsen D, Coomans D. Intravesical chemotherapy or immunotherapy for intermediate-risk non-muscle invasive bladder cancer: does the patient mention a different quality of life? Urology 2013;82:S130-1.

\section{EDITORIAL COMMENT}

\section{Invitation to Cochrane Urology}

January 1, 2020 marked the formal launch of the Korean Satellite of Cochrane Urology. The purpose of this Satellite is to promote the development and dissemination of high-quality systematic reviews to guide evidence-based clinical practice in urology, especially in Korea and other AsiaPacific countries. It will also provide editorial support for Cochrane authors and work closely with Cochrane Korea to provide systematic review training. The resulting Cochrane reviews will be published in the Cochrane Library, but those of greatest interest will also be co-published in Investigative and Clinical Urology (ICUrology), in an abbreviated and condensed version meant to meet the information needs of busy clinicians all around the world. This abridged Cochrane Review is the first co-publication in ICUrology.

To provide further background information about Cochrane and Urology and its activities: Cochrane Urology (https://urology.cochrane.org/) is part of the greater Cochrane organization (https://www.cochrane.org/), an international not-for-profit organization founded in 1993 to systematically assess what works (and what does not) in health care. Within Cochrane, a global network of volunteer authors and contributors seek to systematically gather, critically appraise, and summarize clinical research evidence to guide physicians, patients, and policy makers in making informed, evidence-based choices about preventive and therapeutic interventions, diagnostic tests, and prognosis of medical conditions. The production of each of these summaries follows a rigorous development process, with several stages of internal and external clinical and methodological peer review. In addition, Cochrane employs a rigorous process for managing real and potential conflicts of interests. As a result, Cochrane reviews are among the highest quality and most trustworthy sources of evidence summaries, and are greatly valued, not only by individual healthcare providers, but also guideline developers and policy makers. There are currently over 7,500 Cochrane Systematic Reviews published in the Cochrane Library (https://www.cochranelibrary.com).

The scope of Cochrane Urology excludes the area of urinary incontinence (which is part of the scope of the Incontinence Group), but otherwise covers the entire breadth of urology, including all of urological oncology, male lower urinary tract symptoms and men's health, as well as stone disease.

Not only is the creation of the Korean Satellite of Cochrane Urology expected to increase review production, but it will also provide a new point of contact for individuals and author teams who are interested in getting involved, especially those from South Korea and surrounding countries.

It is our dedicated goal to engage more urologists, nurses, and other healthcare professionals in our field from this part of the world to address the most pressing clinical questions through high-quality systematic reviews. To do so, po- 
tential contributors should reach out to the Editorial Group (cochraneurology_korea@yonsei.ac.kr) to discuss ideas and interests. Aside from becoming a Cochrane author, there are a number of other opportunities to become involved such as being a consumer representative, helping with the translation and screening of foreign language studies. We explicitly welcome all members of the Korean Urological Association into the Cochrane ecosystem. It offers great promise for collaboration and resource-sharing and should serve the Korean Urological Association in its efforts to promote highquality, evidence-based care.

\section{CONFLICTS OF INTEREST}

The authors have nothing to disclose.

Jae Hung Jung and Eu Chang Hwang are Contact Editors of Cochrane Urology, Philipp Dahm serves as Coordinating Editor of Cochrane Urology for the international Cochrane Collaboration.

\section{ACKNOWLEDGMENTS}

We are very grateful to acknowledge the support received from Sang-Baek Koh, Professor, Department of Preventive Medicine, Yonsei University Wonju College of Medicine, Wonju, for his support of the Korean Satellite of Cochrane Urology.

\section{AUTHORS' CONTRIBUTIONS}

Research conception and design: Jae Hung Jung and Philipp Dahm. Data acquisition, statistical analysis, data analysis and interpretation, obtaining funding, administrative, technical, or material support: not available. Drafting of the manuscript: Jae Hung Jung and Eu Chang Hwang. Critical revision of the manuscript: Philipp Dahm. Approval of the final manuscript: Philipp Dahm.

$$
\text { Jae Hung Jung }{ }^{1,2} \text { (1), Eu Chang Hwang }{ }^{2,3} \text { (1), }
$$
Philipp Dahm ${ }^{4,5}$ (i)

${ }^{1}$ Department of Urology, Yonsei University Wonju College of Medicine, Wonju, ${ }^{2}$ Institute of Evidence-based Medicine, Yonsei University Wonju College of Medicine, Wonju, ${ }^{3}$ Department of Urology, Chonnam National University Medical School, Gwangju, Korea, ${ }^{4}$ Department of Urology, University of Minnesota, Minneapolis, MN, ${ }^{5}$ Urology Section, Minneapolis VA Health Care System, Minneapolis, MN, USA Corresponding Author: Philipp Dahm Minneapolis VA Health Care System, Urology Section 112D, 1 Veterans Drive, Minneapolis, MN 55417, USA TEL: +1-612-467-3532, FAX: +1-612-467-2232, E-mail: pdahm@umn.edu ORCID: https:/orcid.org/0000-0003-2819-2553 